\title{
Inventário das espécies de Cassidinae (Insecta, Coleoptera, Chrysomelidae) do Parque Nacional do Itatiaia, RJ, Brasil
}

\author{
Marianna Vieira dos Passos Simões ${ }^{1,2}$ \& Marcela Laura Monné ${ }^{1}$ \\ IDepartamento de Entomologia, Museu Nacional, Universidade Federal do Rio de Janeiro - UFRJ, \\ Quinta da Boa Vista, São Cristóvão, CEP 20940-040, Rio de Janeiro, RJ, Brasil \\ ${ }^{2}$ Autor para correspondência: Marianna Vieira dos Passos Simões, e-mail: mari15_2@hotmail.com
}

SIMÕES, M.V.P. \& MONNÉ, M.L. Inventory of the Cassidinae species (Insecta, Coleoptera, Chrysomelidae) of the Parque Nacional do Itatiaia, RJ, Brazil. Biota Neotrop. 11 (4): http://www.biotaneotropica.org.br/v11n4/ en/abstract?inventory+bn01811042011

\begin{abstract}
A survey of the Cassidinae species recorded to the Parque Nacional do Itatiaia, state of Rio de Janeiro, is presented. The Parque Nacional do Itatiaia (PNI), is situated in the state of Rio de Janeiro, Brazil and is one of the most important Mata Atlântica Conservation Unit. Little is known about the species of Coleoptera from this park. Two surveys concerning Cassidinae fauna of Rio de Janeiro were previously done, totaling 80 species recorded to PNI. The main goal of this study is to update the checklist of species as well as to provide an identification key of Cassidinae tribes that occur at the PNI. We examined specimens from the collection of Museu Nacional, Universidade Federal do Rio de Janeiro and Fundação Instituto Oswaldo Cruz. In addition, samples were done from september, 2007 to january, 2010.This study resulted in 88 species distributed in 29 genera and seven tribes. Nineteen species have now new records of distribution, eight of them for the state of Rio de Janeiro, Parque Nacional do Itatiaia.
\end{abstract}

Keywords: Cassidinae, inventory, identification key, new records, Atlantic Rainforest.

SIMÕES, M.V.P. \& MONNÉ, M.L. Inventário das espécies de Cassidinae (Insecta, Coleoptera, Chrysomelidae) do Parque Nacional do Itatiaia, RJ, Brasil. Biota Neotrop. 11(4): http://www.biotaneotropica.org.br/v11n4/pt/ abstract?inventory+bn01811042011

Resumo: É apresentado um levantamento das espécies de Cassidinae que ocorrem no Parque Nacional do Itatiaia, Rio de Janeiro. O Parque Nacional do Itatiaia (PNI) é situado no Estado do Rio de Janeiro, Brasil e é uma das mais importantes Unidades de Conservação da Mata Atlântica. Pouco se sabe sobre as espécies de Coleoptera desse parque. Dois inventários de Cassidinae foram realizados previamente no Estado do Rio de Janeiro, totalizando 80 espécies para o PNI. O objetivo do trabalho é realizar uma atualização do inventário das espécies e fornecer uma chave de identificação para as tribos de Cassidinae que ocorrem no parque. Foram examinados exemplares da coleção do Museu Nacional, Universidade Federal do Rio de Janeiro e da Fundação Instituto Oswaldo Cruz. Em adição, foram realizadas coletas no período de setembro de 2007 a janeiro de 2010 . Este estudo resultou em 88 espécies distribuídas em 29 gêneros e sete tribos. Dezenove apresentam novos registros de distribuição sendo que destes, oito são para o Estado do Rio de Janeiro, Parque Nacional do Itatiaia.

Palavras-chave: Cassidinae, inventário, chave de identificação, novos registros, Mata Atlântica. 


\section{Introdução}

Chrysomelidae compreende 19 subfamílias e cerca de 37.000 espécies (Chaboo 2007). Possui distribuição mundial com maior número de espécies nos trópicos, principalmente na América do Sul (Borowiec \& Świetojanska 2010). Cassidinae Gyllenhal, 1813, segunda maior subfamília em número de espécies depois de Galerucinae, apresenta 6.000 espécies, com cerca de 1.000 registradas no Brasil. São comumente chamados de "tortoise beetles" na América do Norte, pois apresentam corpo com contorno ovalado ou arredondado, geralmente distintamente convexo dorsalmente e achatado ventralmente e com as margens dos élitros e pronoto achatadas e dilatadas (Costa Lima 1955, Chaboo 2007).

Foram realizados dois inventários em Cassidinae, que incluiram o Parque Nacional do Itatiaia, são eles: Zikán \& Zikán (1967) que registraram 20 espécies no Itatiaia e Serra da Mantiqueira e Flinte et al. (2009) que registraram 188 espécies no Estado do Rio de Janeiro, adicionando 60 espécies para o PNI, totalizando 80 espécies para o PNI e 174 para o Estado do Rio de Janeiro.

Este trabalho tem como objetivo atualizar o inventário das espécies de Cassidinae que ocorrem no parque com base em dados da literatura, da coleção de Cassidinae do Museu Nacional, Universidade Federal do Rio de Janeiro (MNRJ), da Fundação Instituto Oswaldo Cruz (FIOCRUZ) e de coletas de campo. Com isso pretende-se fornecer dados de distribuição para as espécies e, eventualmente, novos registros e fornecer uma chave de identificação para as tribos de Cassidinae do PNI.

\section{Material e Métodos}

O PNI situa-se entre os paralelos $22^{\circ} 19^{\prime}$ e $22^{\circ} 45^{\prime}$ latitude Sul e os meridianos $44^{\circ} 15^{\prime}$ e $44^{\circ} 50^{\prime}$ de longitude Oeste. Está localizado na divisa entre os estados do Rio de Janeiro, Minas Gerais e São Paulo, na Serra da Mantiqueira. Fica ao sudoeste do Estado do Rio de Janeiro, no município de Itatiaia, e a sudoeste do Estado de Minas gerais, abrangendo os Municípios de Itamonte, Alagoas e Bocaina de Minas (IBAMA 2010).

Foram estudados os exemplares da coleção do MNRJ, FIOCRUZ com registro para o Parque Nacional do Itatiaia. Além disso, foram realizadas 10 coletas com guarda-chuva entomológico e rede entomológica, entre setembro de 2007 e janeiro de 2010 (Licença de coleta do IBAMA n ${ }^{\circ}$ 16928-1) próximas à sede do parque, em altitudes de 700 a $1250 \mathrm{~m}$. O material coletado foi montado, etiquetado e depositado no MNRJ.

A lista de espécies é apresentada em ordem alfabética de tribo, gênero e espécie e as referências sob cada espécie restringem-se à descrição original e às dos autores que citaram a espécie para o parque. O item distribuição de cada espécie foi obtido em Borowiec \& Świetojanska (2010). O item material examinado foi incluído quando ocorre um novo registro. As espécies obtidas nas coletas são destacadas com um asterisco ao lado do nome da espécie.

\section{Resultados}

No PNI foram encontradas 88 espécies distribuídas em sete tribos: Cassidini Gyllenhal, 1813; Dorynotini Monrós \& Viana, 1949; Goniocheniini Spaeth, 1942; Imatidiini Chapuis, 1875; Omocerini Hincks, 1952; Spilophorini Chapuis, 1875; Stolaini Hincks, 1952. Para 19 espécies são fornecidos novos registros de distribuição sendo que destes, oito são para o Estado do Rio de Janeiro, no PNI.

Chave para as tribos de Cassidinae do Parque Nacional do Itatiaia, RJ:

1. Antena com 10 antenômeros Imatidiini
1'. Antena com 11 antenômeros .2

2(1). Garras tarsais apendiculadas. Stolaini

2'. Garras tarsais simples ou pectinadas.. 3

3(2’). Antenômeros III-VI aparentemente fusionados; metatrocanter com projeção na margem ventral; antenas inseridas em uma única cavidade antenal (esternito I e II fusionados e com sutura vestigial) (Calyptocephala nigricornis Germar, 1824).

3'. Antenômeros basais visivelmente segmentados, metatrocanter sem projeção na margem ventral; cavidade antenal dupla.....

4(3’). Escutelo em forma de losango; élitros (em vista lateral) com espinho longo, aprox. 2/3 da largura dos élitros com ângulo ânterolateral projetados anteriormente, alcançando a metade do pronoto e com carena oblíqua até o ângulo umeral. Dorynotini

4'. Escutelo com outro aspecto; élitros sem projeções, com uma projeção curta ou com duas projeções .....................................................

5(4'). Margem anterior do protórax arredondada (cabeça não visível em vista dorsal). .Cassidini

5'. Margem anterior do protórax sinuosa . .6

6(5'). Colar prosternal bem desenvolvido e recobre parcialmente as peças bucais; protórax subtrapezoidal ou arredondado..........Omocerini

6'. Colar prosternal não recobre as peças bucais; protórax transverso, lados paralelos...................................................... Goniocheniini

\section{Tribo Cassidini Gyllenhal, 1813}

Segundo Chaboo (2007) Cassidini apresenta 76 gêneros e 964 espécies e no PNI foram encontradas 37 espécies em 11 gêneros. Seis espécies apresentaram novas ocorrências de distribuição: três no Rio de Janeiro (PNI), uma em Minas Gerais, uma em Santa Catarina e uma no Espírito Santo.

1. Agroiconota judaica (Fabricius, 1781) (Figura 1)

Cassida iudaica Fabricius 1781: 109.

Agroiconota judaica: Flinte et al. 2009: 574.

Distribuição-Nicarágua; Costa Rica; Panamá, Colômbia, Trinidade e Tobago, Venezuela, Guiana, Suriname, Guiana Francesa, Brasil (Amazonas, Pará, Maranhão, Rondônia, Mato Grosso, Pernambuco, Distrito Federal, Rio Grande do Sul), Equador, Peru, Bolívia, e Paraguai. Amplia-se a distribuição para o Estado de Minas Gerais.

Material examinado. BRASIL, Minas Gerais: 1 exemplar, Oscar Monte col. (MNRJ).

\section{Agroiconota pullula (Boheman 1855)}

Copotocycla pullula Boheman 1855: 359.

Agroiconota pullula: Flinte et al. 2009: 574.

Distribuição-Brasil (Minas Gerais, Rio de Janeiro, São

Paulo) Peru, Bolívia e Paraguai.

3. Charidotella (Charidotella) incerta (Boheman, 1855)*(Figura 2)

Coptocycla incerta Boheman 1855: 191. 
Charidotella (Charidotella) incerta: Flinte et al. 2009: 576.

Distribuição-Brasil (Minas Gerais, Rio de Janeiro, São Paulo).

4. Charidotella (Charidotella) pellucida (Boheman, 1855)

Coptocycla pellucida Boheman 1855: 343.

Charidotella (Charidotella) pellucida: Flinte et al. 2009: 577.
Distribuição-Brasil (Rio de Janeiro), Paraguai e Argentina.

5. Charidotella (Charidotella) recidiva (Spaeth1926)*

Metriona recidiva Spaeth 926: 23.

Charidotella (Charidotella) recidiva: Flinte et al. 2009: 577.

Distribuição-Brasil (Minas Gerais, Rio de Janeiro, São

Paulo, Santa Catarina, Rio Grande do Sul) e Argentina.
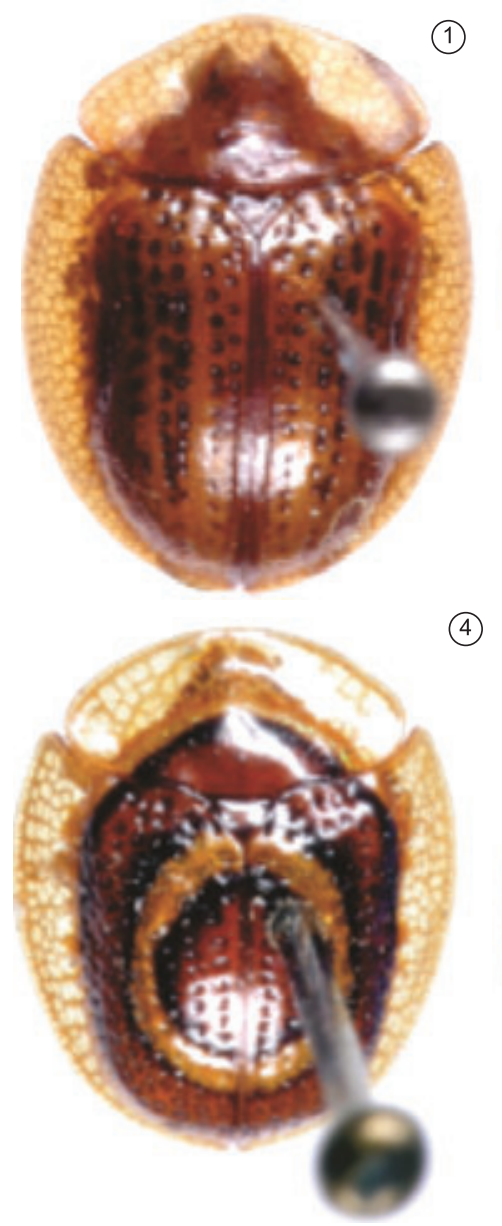

(7)

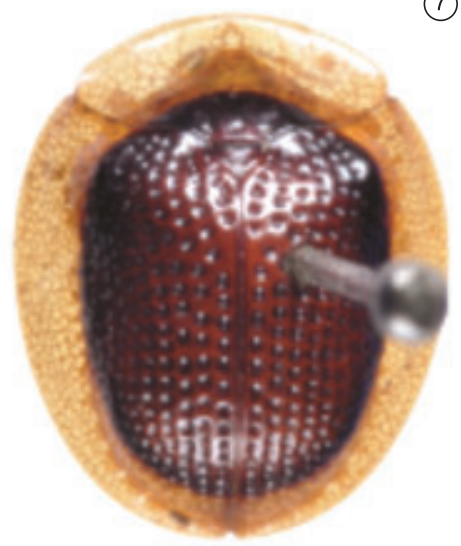

(2)

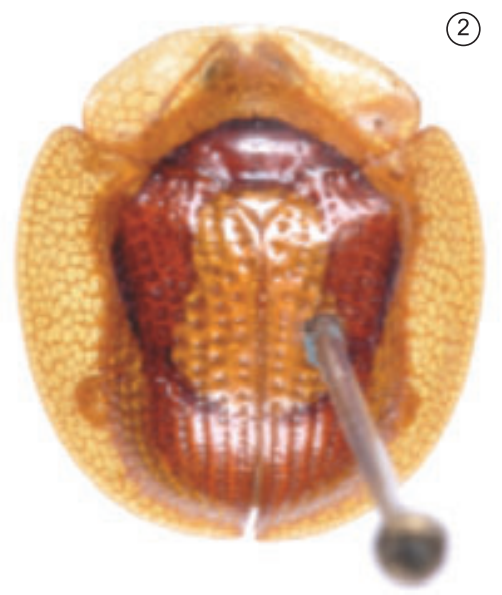

(5)
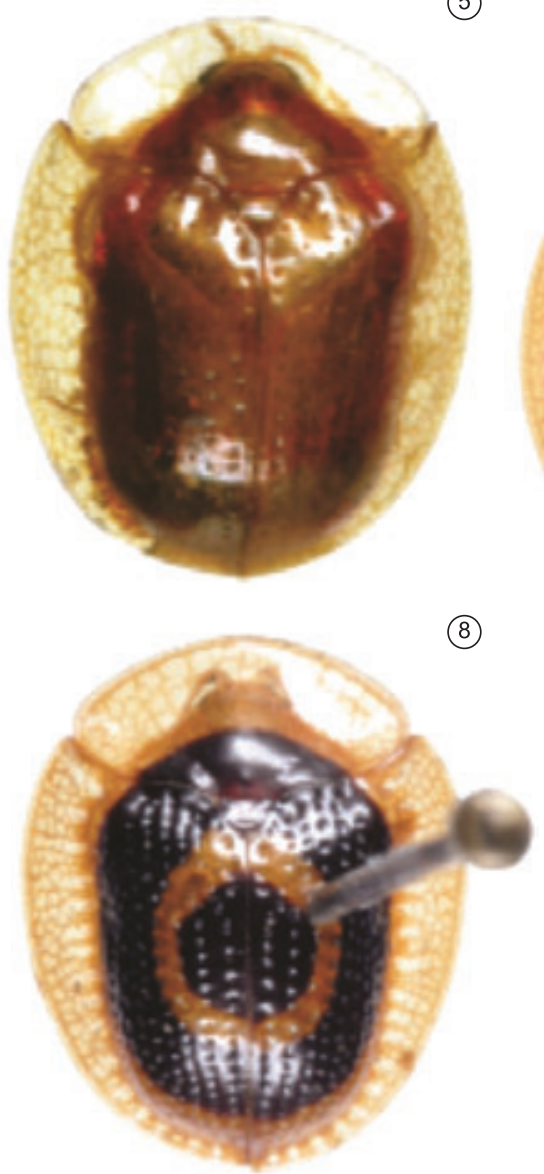

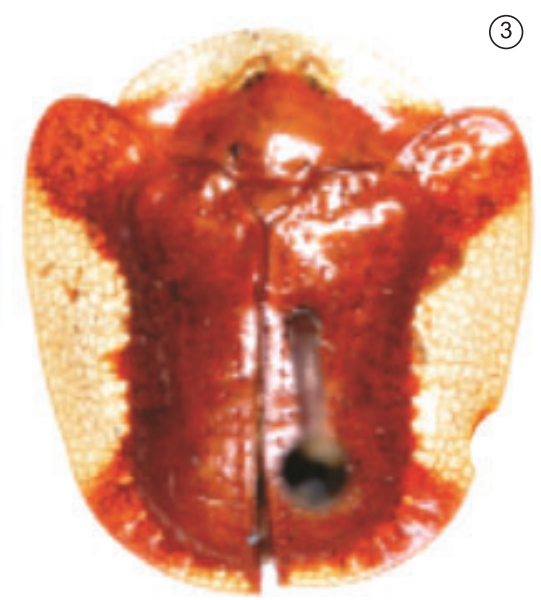

(6)

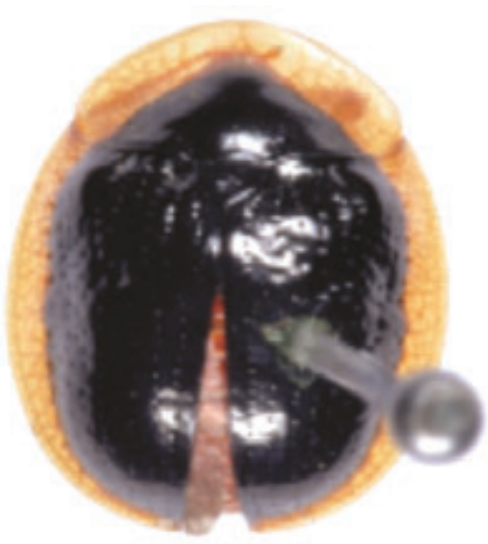

(9)

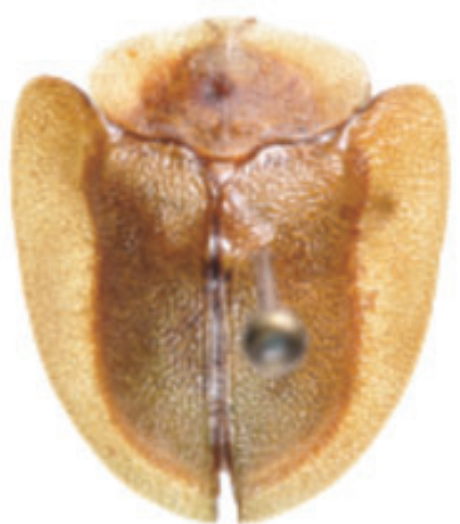

Figuras 1-9. 1) Agroiconota judaica; 2) Charidotella incerta; 3) Charidotella rubincunda; 4) Charidotis annularis; 5) Charidotis biarcuata; 6) Charidotis circuncripta; 7) Charidotis furunculus; 8) Charidotis incincta; 9) Metriona elatior.

Figures 1-9. 1) Agroiconota judaica; 2) Charidotella incerta; 3) Charidotella rubincunda; 4) Charidotis annularis; 5) Charidotis biarcuata; 6) Charidotis circuncripta; 7) Charidotis furunculus; 8) Charidotis incincta; 9) Metriona elatior. 
6. Charidotella (Metrionaspis) rubicunda (Guérin-Méneville 1844) (Figura 3)

Aspidomorpha rubicunda Guérin-Méneville 1844: 291.

Charidotella (Metrionaspis) rubicunda: Flinte et al. 2009: 578.

Distribuição-Equador, Brasil (Amazonas, Mato Grosso,

Bahia, Minas Gerais, Rio de Janeiro, São Paulo, Paraná), Peru, Bolívia, Paraguai e Argentina.

7. Charidotis annularis (Boheman, 1855) (Figura 4)

Coptocycla annularis Boheman 1855: 207.

Charidotis annularis: Zikán \& Zikán 1967: 143, Flinte et al. 2009: 578.

Distribuição-Brasil (Rio de Janeiro).

8. Charidotis biarcuata (Boheman, 1855)* (Figura 5)

Coptocycla biarcuata Boheman 1855: 274.

Charidotis biarcuata: Flinte et al. 2009: 578.

Distribuição-Brasil (Rio de Janeiro).

9. Charidotis circumducta (Boheman, 1855)

Coptocycla circumducta Boheman 1855: 161.

Charidotis circumducta: Flinte et al. 2009: 579.

Distribuição-Panamá e Brasil (Rio de Janeiro, São Paulo, Santa Catarina, Rio Grande do Sul).

10. Charidotis circumscripta (Boheman, 1855) (Figura 6)

Charidotis circumscripta Boheman 855: 24.

Charidotis circumscripta: Zikán \& Zikán 1967: 143, Flinte et al. 2009: 579.

Distribuição-Brasil (São Paulo, Santa Catarina, Rio Grande do Sul) e Panamá.

11. Charidotis concentrica (Boheman, 1855)

Coptocycla concentrica Boheman 1855: 205.

Charidotis concentrica: Flinte et al. 2009: 579.

Distribuição-Brasil (Rio de Janeiro).

12. Charidotis furunculus Boheman, 1855 (Figura 7)

Coptocycla furunculus Boheman 1855: 216.

Charidotis furunculus: Zikán \& Zikán 1967: 143, Flinte et al. 2009: 580.

Distribuição-Brasil (Rio de Janeiro, São Paulo) e Argentina. Amplia-se a distribuição para o Estado de Santa Catarina.

Material examinado. BRASIL, Santa Catarina: Mafra, 1 exemplar, XII.1948, A. Maller col. (MNRJ).

13. Charidotis incincta (Boheman, 1862) (Figura 8)

Coptocycla incincta Boheman 1862: 422.

Distribuição-Costa Rica, Panamá, Venezuela, Brasil (Amazonas, Mato Grosso, Pernambuco) e Bolívia. Amplia-se a distribuição no Brasil para o Estado do Rio de Janeiro.

Material examinado. BRASIL, Rio de Janeiro: Itatiaia
(Parque Nacional do Itatiaia), 1 exemplar, 16.I.1925, J. F. Zikán col. (FIOCRUZ).

14. Charidotis neglecta (Boheman, 1855)

Coptocycla neglecta Boheman 1855: 165.

Charidotis neglecta: Zikán \& Zikán 1967: 144.

Distribuição-Brasil (Rio de Janeiro, São Paulo).

15. Charidotis nucleata (Boheman, 1855)

Charidotis nucleata Boheman 1855: 18.

Charidotis nucleata: Flinte et al. 2009: 580.

Distribuição-Brasil (Rio de Janeiro).

16. Coptocycla (Podostraba) arcuata (Swederus, 1787)*

Cassida arcuata Swederus 1787: 193.

Coptocycla (Podostraba) arcuata: Flinte et al. 2009: 584.

Distribuição-Brasil (Minas Gerais, Rio de Janeiro, São Paulo, Santa Catarina).

17. Ctenocharidotis roseopicta (Boheman, 1855)

Coptocycla roseopicta Boheman 1855: 233.

Ctenocharidotis roseopicta: Flinte et al. 2009: 585.

Distribuição-Brasil (Bahia, Rio de Janeiro).

18. Hybosa gibbera Boheman, 1855*

Hybosa gibbera Boheman 1855: 2.

Hybosa gibbera: Flinte et al. 2009: 588.

Distribuição-Brasil (Rio de Janeiro). Amplia-se a distribuição no Brasil para o Estado do Espírito Santo.

Material examinado. BRASIL, Espírito Santo: Colatina, 1 macho, XI.1970, A. Silva col. (MNRJ).

19. Metriona elatior (Klug 1829) (Figura 9)

Cassida elatior Klug 1829: 8.

Metriona elatior: Flinte et al. 2009: 590.

Distribuição-Guiana Francesa, Brasil (Pará, Goiás, Minas Gerais, Rio de Janeiro ao Rio Grande do Sul), Paraguai, Uruguai e norte da Argentina.

20. Microctenochira aciculata (Boheman, 1855)* (Figura 10)

Coptocycla aciculata Boheman 1855: 465.

Microctenochira aciculata: Zikán \& Zikán 1967: 144, Flinte et al. 2009: 590.

Distribuição-Brasil (Bahia, Rio de Janeiro, São Paulo, Santa Catarina), Paraguai e Argentina.

21. Microctenochira gagatina (Spaeth 1902)

Ctenochira gagatina Spaeth 1902: 94.

Microctenochira gagatina: Flinte et al. 2009: 590.

Distribuição-Brasil (Rio de Janeiro).

22. Microctenochira optata (Boheman, 1855)*(Figura 11)

Coptocycla optata Boheman 1855: 470. 
Microctenochira optata: Flinte et al. 2009: 591.

Distribuição-Guiana Francesa, Brasil (Pará, Alagoas, Bahia, Mato Grosso, Minas Gerais, Rio de Janeiro ao Rio Grande do Sul), Peru, Paraguai e Argentina.

23. Microctenochira patruelis (Boheman, 1855)

Coptocycla patruelis Boheman 1855: 467.

Microctenochira patruelis: Flinte et al. 2009: 591.

Distribuição-Brasil (Goiás, Minas Gerais, Espírito Santo, Rio de Janeiro).

24. Microctenochira stigmatica (Boheman, 1855) (Figura 12)

Coptocycla stigmatica Boheman 1855: 476.

Microctenochira stigmatica: Flinte et al. 2009: 591.

Distribuição-Brasil (Bahia, Paraná ao Rio Grande do Sul).

25. Microctenochira villica (Boheman, 1855)* (Figura 13)

Coptocycla villica: Boheman 1855: 217.

Distribuição-"Brasilia". Registra-se a distribuição no Brasil para o Estado do Rio de Janeiro.

Material examinado. BRASIL, Rio de Janeiro: Itatiaia (Parque Nacional do Itatiaia), 1100 m, 1 exemplar, 28.

IX.2007, C. Patiu, J. Mermudes, M. L. Monné \& M. A. Monné col. (MNRJ).

26. Orexita picta (Boheman, 1855)*(Figuras 14,15)

Coptocycla picta Boheman 1855: 94.

Orexita picta: Flinte et al. 2009: 593

Distribuição-Argentina, Brasil (Rio de Janeiro, São Paulo, Rio Grande do Sul) e Paraguai.

27. Plagiometriona ambigena (Boheman, 1855)*(Figura 17)

Coptocycla ambigena Boheman 1855: 248.

Plagiometriona ambigena: Flinte et al. 2009: 595.

Distribuição-Brasil (Rio de Janeiro, Santa Catarina).

28. Plagiometriona clandestina (Boheman, 1855)*(Figura 18)

Coptocycla clandestina: Boheman 1855: 370.

Distribuição-Brasil (Santa Catarina). Amplia-se a distribuição no Brasil para o Estado do Rio de Janeiro.

Material examinado. BRASIL, Rio de Janeiro: Itatiaia (Parque Nacional do Itatiaia), 800 m, 1 exemplar, 25.XI.1974, H. S. \& M. A. Monné col. (MNRJ).

29. Plagiometriona dodonea (Boheman, 1855)

Coptocycla dodonea Boheman 1855: 252.

Plagiometriona dodonea: Flinte et al. 2009: 595.

Distribuição-Brasil (Rio de Janeiro).

30. Plagiometriona dorsosignata (Boheman, 1855)

Coptocycla dorsosignata Boheman 1855: 378.

Plagiometriona dorsosignata: Flinte et al. 2009: 595.

Distribuição-Brasil (Rio de Janeiro, São Paulo).
31. Plagiometriona forcipata (Boheman, 1855)

Coptocycla forcipata Boheman 1855: 198.

Plagiometriona emarcida: Flinte et al. 2009: 595.

Plagiometriona forcipata: Flinte et al. 2009: 595.

Distribuição-Brasil (Rio de Janeiro, São Paulo).

32. Plagiometriona herbea (Boheman, 1855)*

Coptocycla herbea Boheman 1855: 353.

Plagiometriona herbea: Flinte et al. 2009: 596.

Distribuição-Brasil (Mato Grosso, Minas Gerais, Rio de Janeiro, Santa Catarina, Rio Grande do Sul), Bolívia e Paraguai.

33. Plagiometriona inscripta (Boheman, 1855)

Coptocycla inscripta Boheman 1855: 368.

Plagiometriona inscripta: Flinte et al. 2009: 596.

Distribuição-Brasil (Rio de Janeiro).

34. Plagiometriona praecincta (Boheman, 1855) (Figura 19)

Coptocycla praecincta Boheman 1855: 360.

Plagiometriona praecincta: Flinte et al. 2009: 596.

Distribuição-Brasil (Rio de Janeiro, São Paulo).

35. Plagiometriona sahlbergi (Boheman, 1855)*

Coptocycla sahlbergi Boheman 1855: 375.

Plagiometriona sahlbergi: Flinte et al. 2009: 596.

Distribuição-Brasil (Rio de Janeiro, Espírito Santo).

36. Plagiometriona vigens (Boheman, 1855)*

Coptocycla vigens Boheman 1855: 429.

Plagiometriona vigens: Flinte et al. 2009: 597.

Distribuição-Brasil (Minas Gerais, Rio de Janeiro, São

Paulo, Rio Grande do Sul).

37. Syngambria bisinuata (Boheman, 1855)*

Coptocycla bisinuata Boheman 1855: 382.

Syngambria bisinuata: Flinte et al. 2009: 601.

Distribuição geográfica-Brasil (Pará, Mato Grosso, Rio de Janeiro Santa Catarina, Rio Grande do Sul), Bolívia, Paraguai e Argentina.

\section{Tribo Dorynotini Monrós \& Viana, 1949}

Segundo Chaboo (2007), Doynotini apresenta seis gêneros e 47 espécies e no PNI foram encontradas duas espécies de Dorynota Chevrolat, 1837, ambas apresentando novos registros de distribuição.

38. Dorynota (Dorynota) bidens (Fabricius, 1781)* (Figuras 20,21)

Cassida bidens Fabricius 1781: 112.

Dorynota (Dorynota) bidens: Flinte et al. 2009: 587.

Distribuição-Trinidade e Tobago, Brasil (Rio de Janeiro) e 

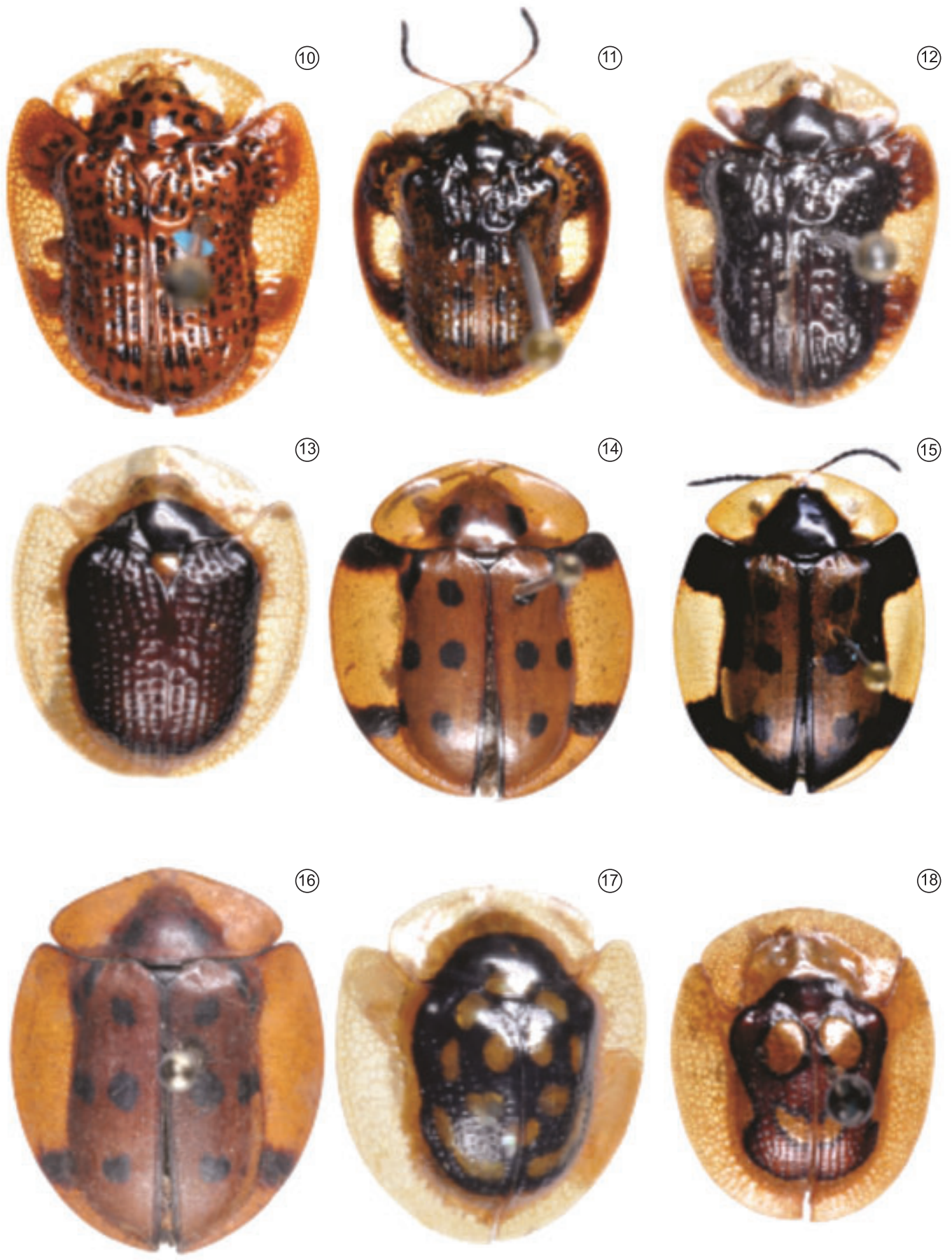

(17)

(18)

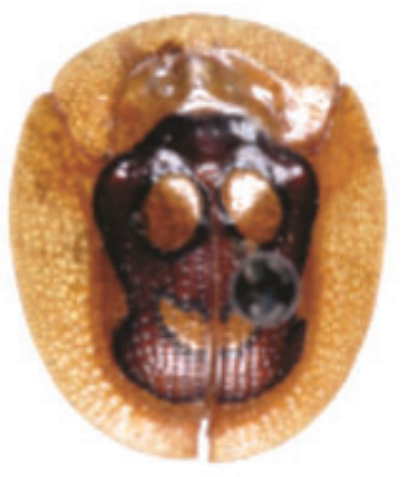

Figuras 10-18. 10) Microctenochira aciculata; 11) Microctenochira optata; 12) Microctenochira stigmatica; 13) Microctenochira villica; 14) Orexita picta; 15) Orexita picta; 16) Orexita picta; 17) Plagiometriona ambigena; 18) Plagiometriona clandestina.

Figures 10-18. 10) Microctenochira aciculata; 11) Microctenochira optata; 12) Microctenochira stigmatica; 13) Microctenochira villica; 14) Orexita picta; 15) Orexita picta; 16) Orexita picta; 17) Plagiometriona ambigena; 18) Plagiometriona clandestina.

Paraguai. Amplia-se a distribuição no Brasil para os Estado do Espírito Santo e São Paulo.

Material examinado. BRASIL, Espírito Santo: Parque

Sooretama, 1 exemplar, XII. 1964, F. M. Oliveira col. (MNRJ);

São Paulo: Engenheiro Coelho, 1 exemplar, 1920, A. Richter col. (MNRJ).
39. Dorynota (Dorynota) monoceros (Germar, 1824)* (Figura 22) Cassida monocerus Germar 1824: 536.

Dorynota (Dorynota) monoceros: Flinte et al. 2009: 587.

Distribuição-Venezuela, Brasil (Rio de Janeiro, Santa Catarina), Bolívia, Paraguai e Argentina. Amplia-se distribuição no Brasil para o Estado de São Paulo. 
Material examinado. BRASIL, São Paulo: Engenheiro Coelho, 1 exemplar, 1920, A. Richter col. (MNRJ).

\section{Tribo Goniocheniini Spaeth, 1942}

Segundo Chaboo (2007), Goniocheniini apresenta cinco gêneros e 14 espécies e no PNI foram encontradas quatro espécies distribuídas em dois gêneros, com um novo registro de distribuição.

40. Chlamydocassis (Ceratocassis) bispinosa (Boheman, 1850)

Desmonota bispinosa Boheman 1850: 154.

Distribuição-Brasil (Minas Gerais, Espírito Santo). Ampliase a distribuição no Brasil para o Estado do Rio de Janeiro.

Material examinado. BRASIL, Rio de Janeiro: Itatiaia

(Parque Nacional do Itatiaia), 1100 m, 1 exemplar, 04.I.1925, J.F. Zikán (FIOCRUZ); Itatiaia, 1100 m, 1 exem-

plar, 20.I.1926, J.F. Zikán (FIOCRUZ).

41. Chlamydocassis retusa (Boheman, 1850)

Desmonota retusa Boheman 1850: 156.

Chlamydocassis retusa: Zikán \& Zikán 1967: 145.

Distribuição-Brasil (Rio de Janeiro).

42. Chlamydocassis (Ceratocassis) subcornuta (Boheman, 1850) (Figuras 23,24)

Desmonota subcornuta Boheman 1850: 155.

Chlamydocassis (Ceratocassis) subcornuta: Flinte et al. 2009: 582. Distribuição-Brasil (Minas Gerais, Rio de Janeiro, São Paulo).

43. Gonochenia (Goniochenia) quadraticollis (Boheman, 1850) (Figura 25)

Mesomphalia quadraticollis Boheman 1850: 219

Gonochenia (Goniochenia) quadraticollis: Zikán \& Zikán 1967: 144, Flinte et al. 2009: 588.

Distribuição-Brasil (Minas Gerais, Espírito Santo, Rio de Janeiro, São Paulo, Santa Catarina).

\section{Tribo Imatidiini Chapuis, 1875}

Segundo Chaboo (2007), Imatidiini apresenta oito gêneros e 78 espécies e no PNI foi encontrada apenas Calliaspis cinnabarina Boheman, 1850, sendo novo registro.

44. Calliaspis cinnabarina (Boheman, 1850) (Figura 26)

Calliaspis cinnabarina Boheman 1850: 84

Distribuição geográfica-Norte do Brasil, Guiana Francesa e

Peru. Registra-se para os estados do Rio de Janeiro e São Paulo.

Material examinado. BRASIL, Rio de Janeiro: Itatiaia

(Parque Nacional do Itatiaia), 1 exemplar, 20.I.1926, J.F.

Zikán (FIOCRUZ); São Paulo: Amparo, 2 exemplares, Paulino

Recch col. (MNRJ).

\section{Tribo Omocerini Hincks, 1952}

Segundo Chaboo (2007) a tribo Omocerini apresenta sete gêneros e 139 espécies e no PNI foram encontradas quatro espécies distribuídas em quatro gêneros e uma nova ocorrência de distribuição.
45. Canistra (Canistrella) rubiginosa (Guérin-Méneville 1844)*

Oxynodera rubiginosa Guérin-Méneville 1844: 289.

Canistra (Canistrella) rubiginosa: Flinte et al. 2009: 575.

Distribuição-Brasil (Goiás, Bahia, Minas Gerais, Espírito

Santo a Santa Catarina), Paraguai, Argentina e Uruguai.

46. Cyclosoma (Dolichotoma) germari (Spaeth, 1913) (Figura 27)

Cassida aenea Germar 1824: 529.

Dolichotoma germari Spaeth 1913: 129.

Cyclossoma (Dolichotoma) germari: Flinte et al. 2009: 585.

Distribuição-Guiana Francesa, Brasil (Amazonas, Minas Gerais, Espírito Santo, Rio de Janeiro, São Paulo, Santa Catarina) e Uruguai.

47. Omocerus (Platytauroma) truncatus (Boheman, 1850) (Figura 28)

Taurom truncata Boheman 1850: 128.

Omocerus (Platytauroma) truncatus: Zikán \& Zikán 1967: 144, Flinte et al. 2009: 593.

Distribuição-Colômbia, Brasil (Amazonas, Minas Gerais ao Rio Grande do Sul), Bolívia, Argentina e Paraguai.

48. Polychalca (Desmonota) platynota (Germar, 1824) (Figura 29)

Cassida platynota Germar 1824: 535.

Polychalca (Desmonota) platynota: Zikán \& Zikán 1967: 145, Flinte et al. 2009: 597.

Distribuição-Brasil (Distrito Federal, Minas Gerais, Rio de Janeiro, São Paulo, Santa Catarina) e Argentina. Registra-se para o Estado do Espírito Santo.

Material examinado. BRASIL, Espírito Santo: Córrego Itá, 1 exemplar, I.1982, H. W. Saer col. (MNRJ).

\section{Tribo Spilophorini Chapuis, 1875}

Segundo Chaboo (2007), Spilophorini apresenta dois gêneros e 30 espécies e no PNI foi encontrada apenas Calyptocephala nigricornis Germar, 1824.

49. Calyptocephala nigricornis Germar, 1824 (Figura 30)

Cassida nigricornis Germar 1824: 540.

Calyptocephala nigricornis: Flinte et al. 2009: 575.

Distribuição-Brasil (Rio de Janeiro, Paraná).

\section{Tribo Stolaini Hincks, 1952}

Segundo Chaboo (2007), Stolaini apresenta gêneros e 528 espécies e no PNI foram encontradas 39 espécies e destas oito apresentam novos registros de distribuição.

50. Acromis spinifex (Linnaeus 1763) (Figuras 31,32)

Cassida spinifex Linnaeus 1763: 392.

Acromis spinifex: Zikán \& Zikán 1967: 143, Flinte et al 2009: 573.

Distribuição-Trinidade e Tobago, Venezuela, Colômbia,

Guiana, Suriname, Guiana Francesa, Brasil, Equador, Peru,

Bolívia, Paraguai e Norte da Argentina. 
(19)

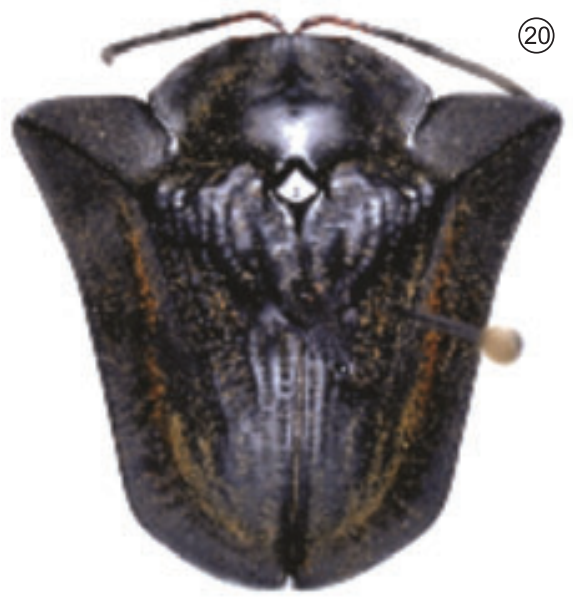

(20)
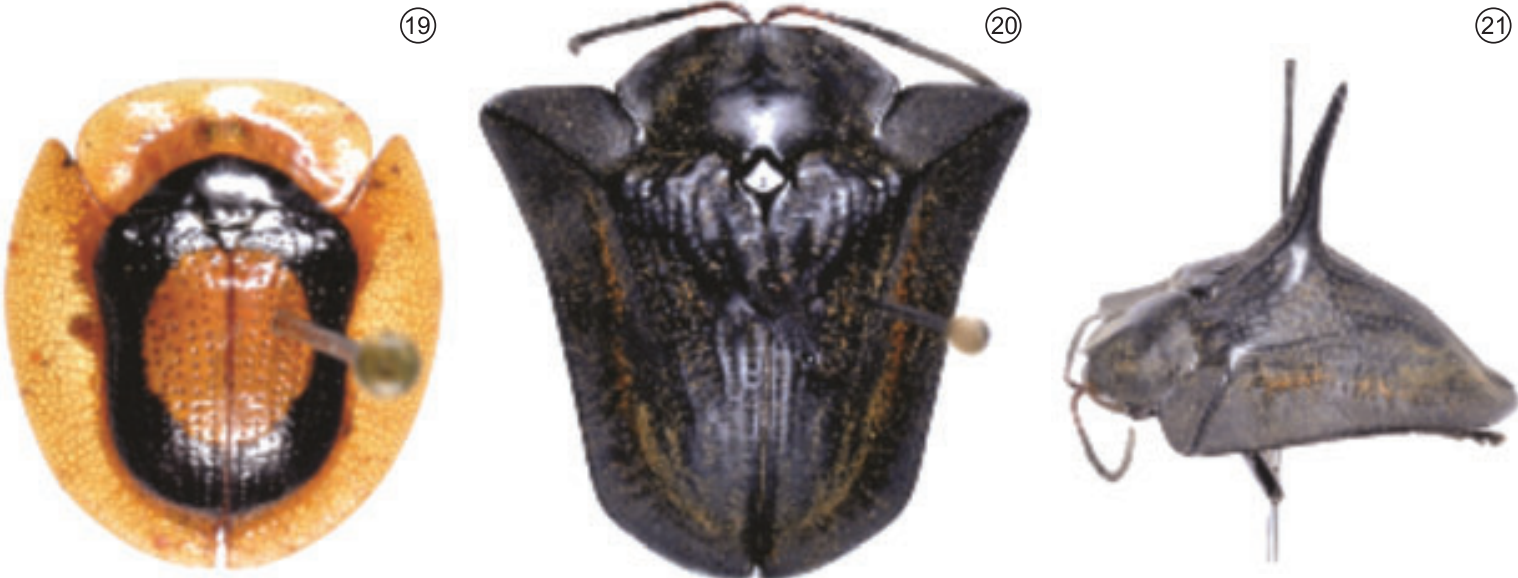

(22)

(23)
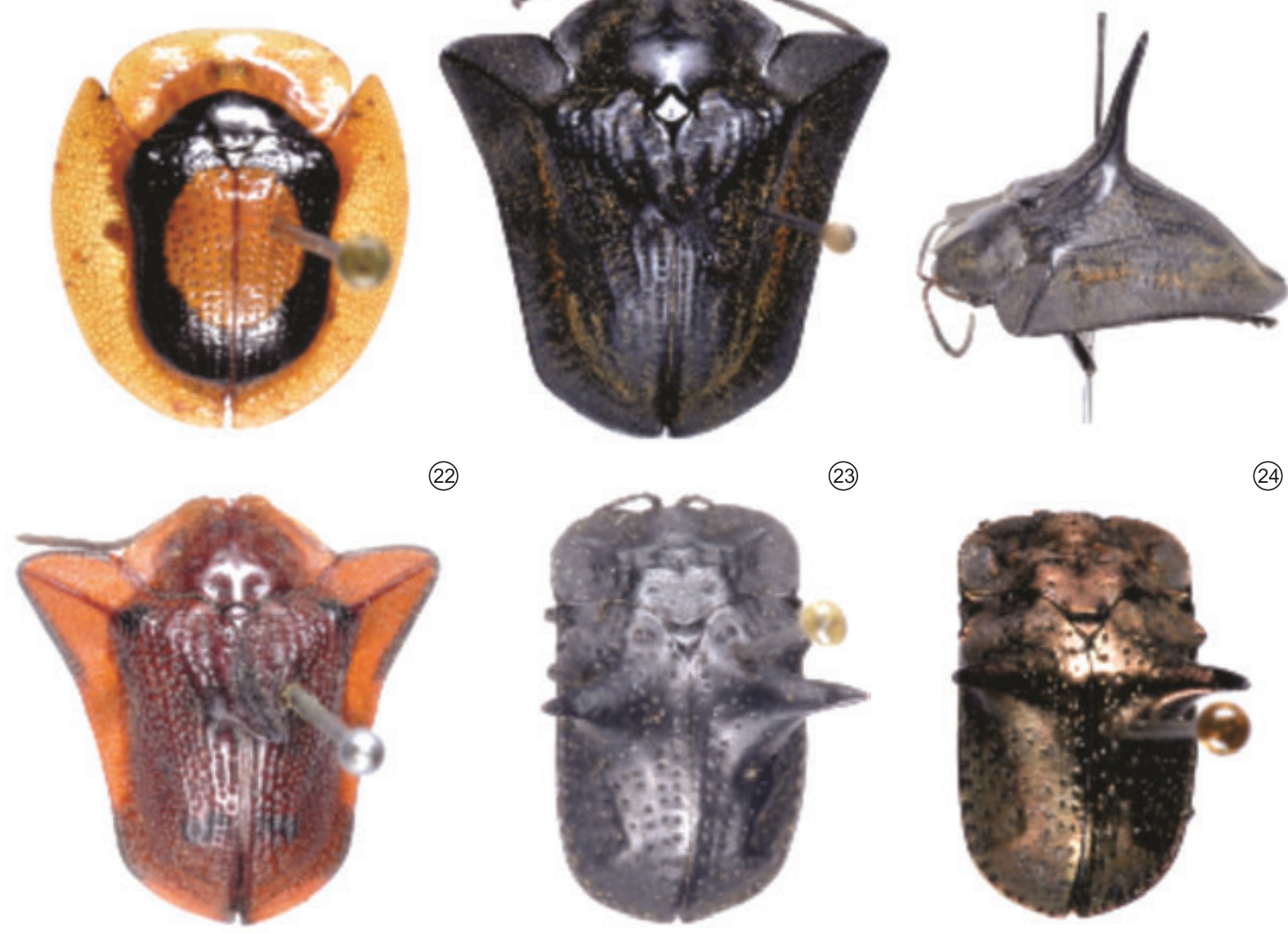

(24)
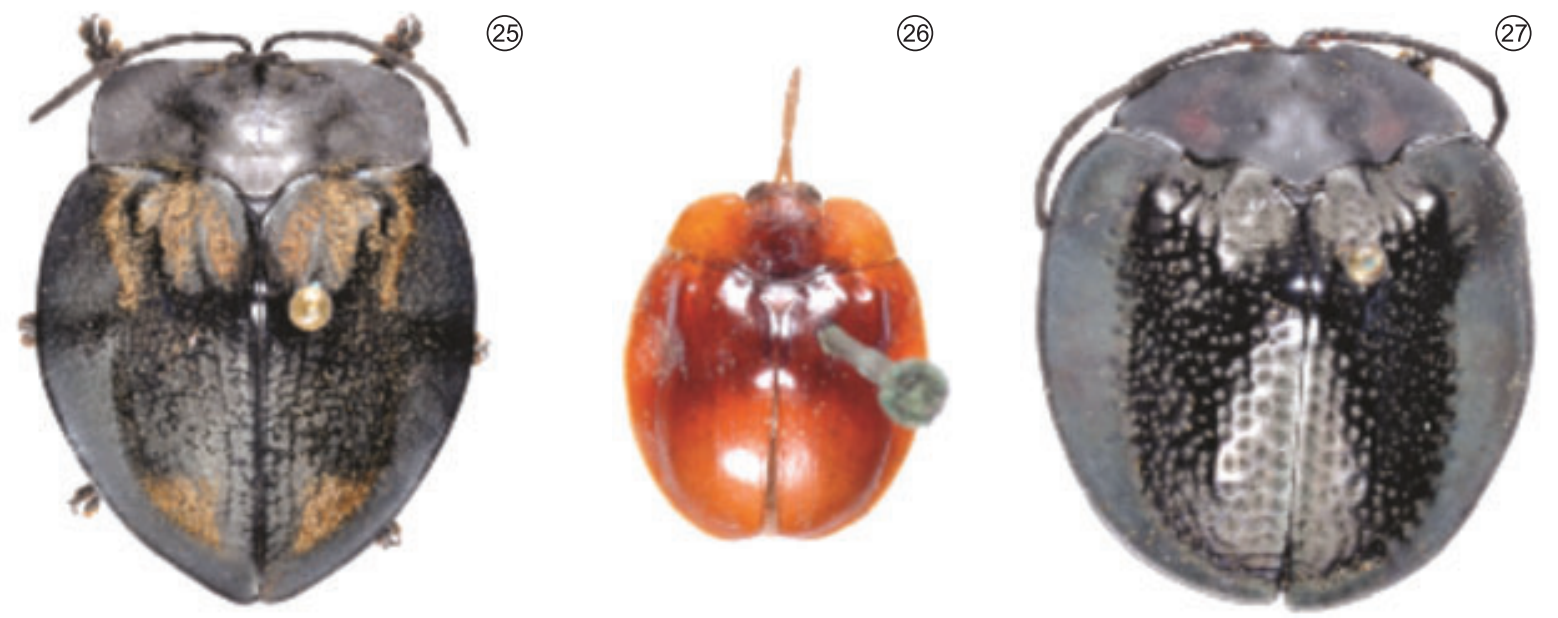

Figuras 19-27. 19) Plagiometriona praecincta; 20) Dorynota bidens; 21) Dorynota bidens (vista lateral); 22) Dorynata monocerus; 23) Chlamydocassis subcornuta; 24) Chlamydocassis subcornuta; 25) Gonochenia quadraticollis; 26) Calliaspis cinnabarina; 27) Cyclossoma germari.

Figures 19-27. 19) Plagiometriona praecincta; 20) Dorynota bidens; 21) Dorynota bidens (vista lateral); 22) Dorynata monocerus; 23) Chlamydocassis subcornuta; 24) Chlamydocassis subcornuta; 25) Gonochenia quadraticollis; 26) Calliaspis cinnabarina; 27) Cyclossoma germari.

\section{Anacassis dubia (Boheman, 1854)* (Figura 33)}

Physonota dubia Boheman 1854: 235.

Anacassis dubia: Flinte et al. 2009: 574.

Distribuição-Brasil (Rio de Janeiro ao Rio Grande do Sul). Registra-se para o Estado de Minas Gerais.
Material examinado. BRASIL, Minas Gerais: Passa Quatro, 1 exemplar, 27.XII.1917, Zikán col. (MNRJ).

52. Anacassis fuscata (Klug 1829) (Figuras 34-37)

Cassida fuscata Klug 1829: 8.

Anacassis fuscata: Flinte et al. 2009: 574. 


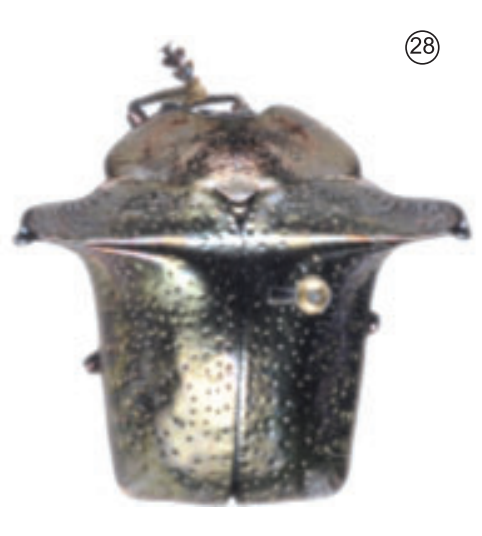

(31)

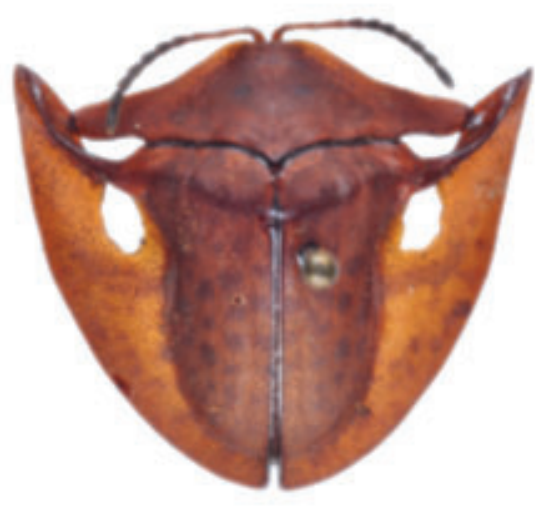

(34)

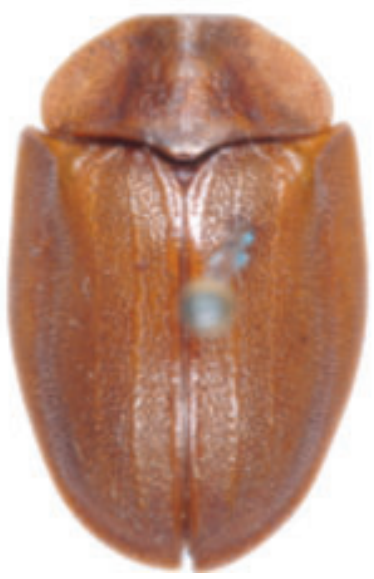

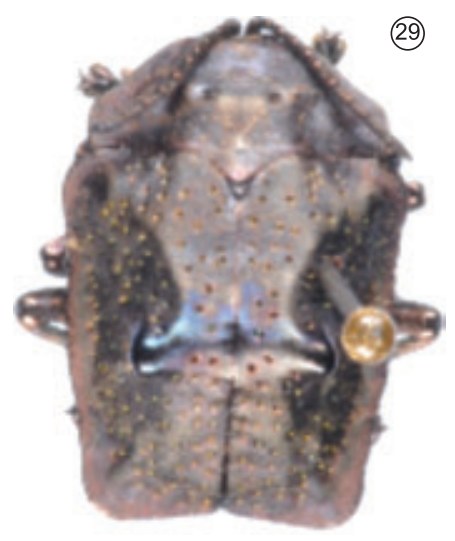

(32)

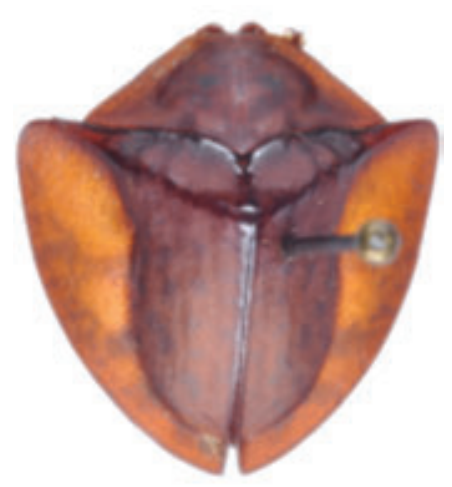

(35)

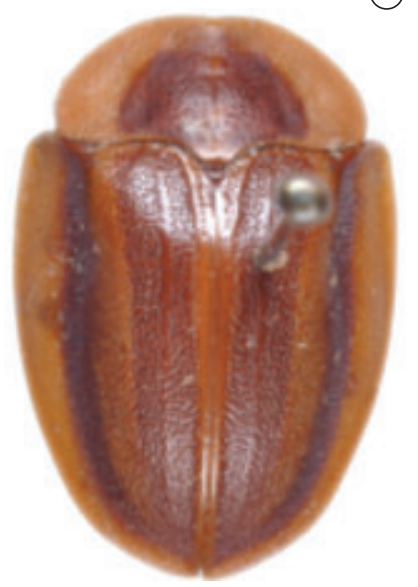

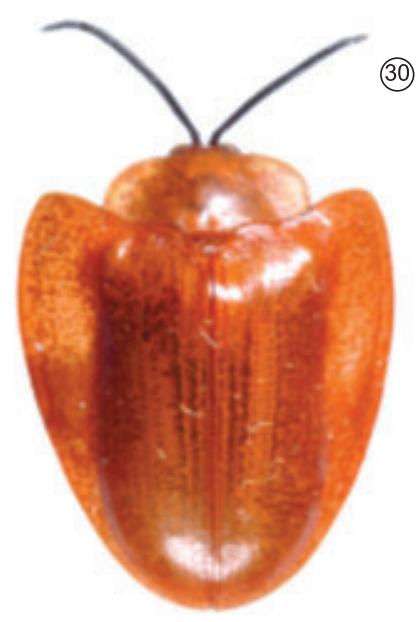

(33)
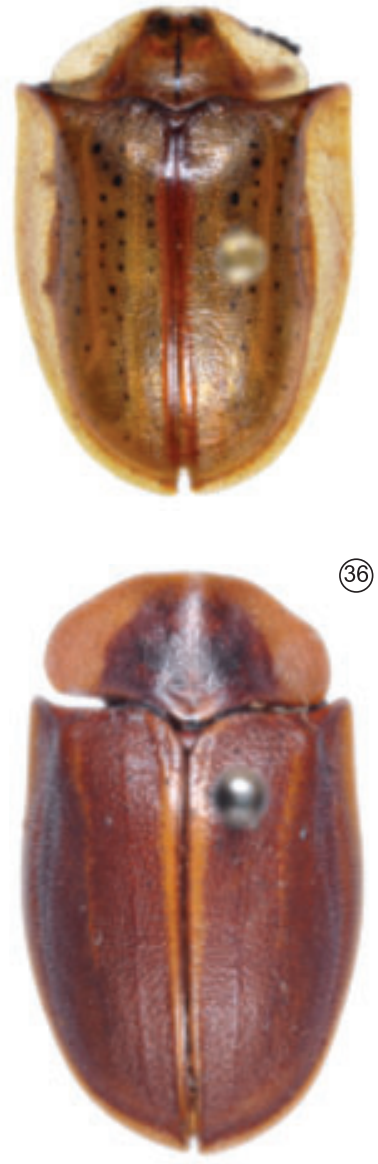

Figuras 28-36. 28) Omocerus truncatus, 29) Polychalca platynota; 30) Calyptocephala nigricornis; 31) Acromis spinifex (ঐ); 32) Acromis spinifex (†); 33) Anacassis dubia; 34) Anacassis fuscatta; 35) Anacassis fuscatta; 36) Anacassis Fuscatta.

Figures 28-36. 28) Omocerus truncatus, 29) Polychalca platynota; 30) Calyptocephala nigricornis; 31) Acromis spinifex (đ)); 32) Acromis spinifex (ㅇ); 33) Anacassis dubia; 34) Anacassis fuscatta; 35) Anacassis fuscatta; 36) Anacassis Fuscatta.

Distribuição-Brasil (Minas Gerais, Rio de Janeiro, São Paulo, Paraná, Santa Catarina, Rio Grande do Sul), Bolívia, Paraguai, Argentina e Uruguai. Novos registros para Argentina (Loreto e Tucumán).

Material examinado. ARGENTINA, Loreto: 1 exemplar, Seabra col. (MNRJ); Tucumán, 1 exemplar, II.1953, Seabra col. (MNRJ).
53. Anacassis punctulata (Klug 1829)

Cassida punctulata Klug 1829: 8.

Anacassis punctulata: Flinte et al. 2009: 575

Distribuição-Brasil (Minas Gerais, Rio de Janeiro, São

Paulo, Paraná, Santa Catarina) e Argentina. 


\section{Botanochara impressa (Panzer 1798) (Figura 38)}

Cassida impressa Panzer 1798: 80.

Distribuição-Brasil (Amapá, Amazonas, Goiás, Maranhão, Minas Gerais, Pará, Paraná, Santa Catarina, São Paulo), Peru, Bolívia, Paraguai e Argentina. Registra-se para o Estado do Rio de Janeiro.

Material examinado. BRASIL, Rio de Janeiro: Itatiaia (Parque Nacional do Itatiaia), 1 exemplar, 30.X.1931 (MNRJ).

55. Botanochara tessellata (Burmeister 1870)

Poecilaspis tesselata Burmeister 1870: 276.

Botanochara tessellata: Flinte et al. 2009: 575.

Distribuição geográfica-Brasil (Pará, Ceará, Mato Grosso, Goiás, Rio de Janeiro), Bolívia, Paraguai e Argentina.

56. Chelymorpha cingulata Boheman, 1854

Chelymorpha cingulata Boheman 1854: 44.

Chelymorpha cingulata: Flinte et al. 2009: 581.

Distribuição-Peru e Brasil (Mato Grosso, Espírito Santo, Rio de Janeiro).

57. Chelymorpha clathrata (Spaeth 1909) (Figura 39)

Chelymorpha clathrata Spaeth 1909: 725.

Distribuição-Brasil (Mato Grosso). Amplia-se a distribuição no Brasil para o Estado do Rio de Janeiro.

Material examinado. BRASIL, Rio de Janeiro: Itatiaia (Parque Nacional do Itatiaia), 700 m, 2 exemplares, 24. XI.1923, J. F. Zikán col. (FIOCRUZ).

58. Chelymorpha constellata (Klug 1829)

Chelymorpha constellata Klug 1829: 7.

Chelymorpha constellata: Flinte et al. 2009: 581.

Distribuição geográfica-Guiana Francesa, Brasil (Pará, Distrito Federal, Minas Gerais, Rio de Janeiro, Santa Catarina) e Peru.

59. Chelymorpha cribraria (Fabricius, 1775)

Cassida cribraria Fabricius 1775: 90.

Chelymorpha cribraria: Flinte et al. 2009: 581

Distribuição-Estados Unidos, Costa Rica, Panamá, Venezuela, Colômbia, Suriname, Guiana Francesa, Brasil (amplamente distribuída), Equador, Peru, Bolívia, Paraguai, Argentina e Uruguai.

60. Chelymorpha infecta (Boheman 1854) (Figura 40)

Chelymorpha infecta Boheman 1854: 34.

Chelymorpha infecta: Flinte et al. 2009: 581.

Distribuição-Argentina e Brasil (Rio de Janeiro).

61. Chelymorpha inflata Boheman, 1854

Chelymorpha inflata Boheman 1854: 12.

Chelymorpha inflata: Flinte et al. 2009: 582.

Distribuição-Brasil (Pará, Rio de Janeiro ao Rio Grande do Sul), Equador, Paraguai e Argentina.
62. Chelymorpha marginata (Linnaeus 1758) (Figura 41)

Cassida marginata Linnaeus 1758: 363.

Chelymorpha marginata: Flinte et al. 2009: 582.

Distribuição-Colômbia, Equador, Guiana Francesa, Suriname, Brasil (Amapá, Pará, Pernambuco, Mato Grosso, Rio de Janeiro), Bolívia e Paraguai. Amplia-se a distribuição no Brasil para o Estado de Minas Gerais.

Material examinado. BRASIL, Minas Gerais: Belo Horizonte, 1 exemplar, II.1932, B. Monte col. (MNRJ).

63. Chelymorpha socia (Boheman 1854)

Chelymorpha socia Boheman 1854: 14.

Chelymorpha socia: Flinte et al. 2009: 582.

Distribuição-Brasil (Maranhão, Bahia, Rio de Janeiro).

64. Cyrtonota cyanea (Linnaeus 1758)* (Figura 42)

Cassida cyanea Linnaeus 1758: 364

Cyrtonota cyanea: Flinte et al. 2009: 586.

Distribuição-Brasil (Rio de Janeiro, Santa Catarina) e Peru.

65. Cyrtonota sexpustulata (Fabricius 1781) (Figura 43)

Cassida 6 pustulata Fabricius 1781: 114.

Cyrtonota sexpustulata: Flinte et al. 2009: 586.

Distribuição-Brasil (Distrito Federal, Rio de Janeiro ao Paraná) e Peru.

66. Cyrtonota similata (Boheman 1850) (Figura 44)

Mesomphalia similata Boheman 1850: 265.

Distribuição-"Brasília”. Registra-se para os estados de Minas Gerais e Rio de Janeiro.

Material examinado. BRASIL, Minas Gerais: Manhumirim, 2 exemplares, Zellibor Hauff col. (MNRJ); Rio de Janeiro: Itatiaia (Parque Nacional do Itatiaia), 1 exemplar, 15.II.1942, Wygod col. (MNRJ); Resende, Serrinha do Alambari, 1 exemplar, IX. 2008, U. Caramaschi col. (MNRJ).

67. Cyrtonota thalassina (Boheman 1850)

Mesomphlaia thalassina Boheman 1850: 326.

Cyrtonota thalassina: Flinte et al. 2009: 586.

Distribuição-Brasil (Pará, Minas Gerais ao Rio Grande do Sul), Paraguai e Argentina.

68. Cyrtonota zikani (Spaeth 1932)

Neomphalia zikáni Spaeth 1932: 193.

Cyrtonota zikani: Flinte et al. 2009: 586.

Distribuição-Brasil (Rio de Janeiro, São Paulo, Rio Grande do Sul).

69. Hilarocassis albida (Germar 1824) (Figura 45)

Cassida albida Germar 1824: 539.

Hilarocassis albida: Flinte et al. 2009: 588. 


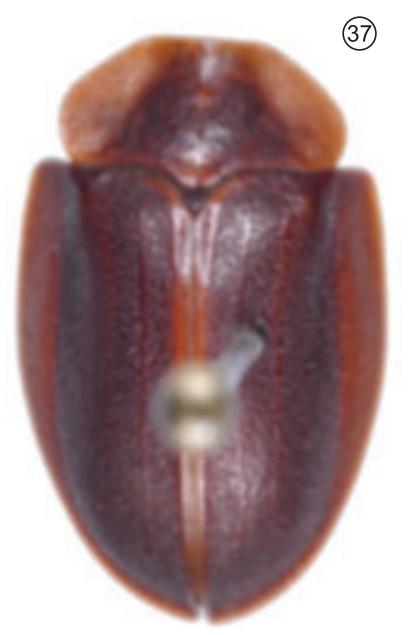

(40)
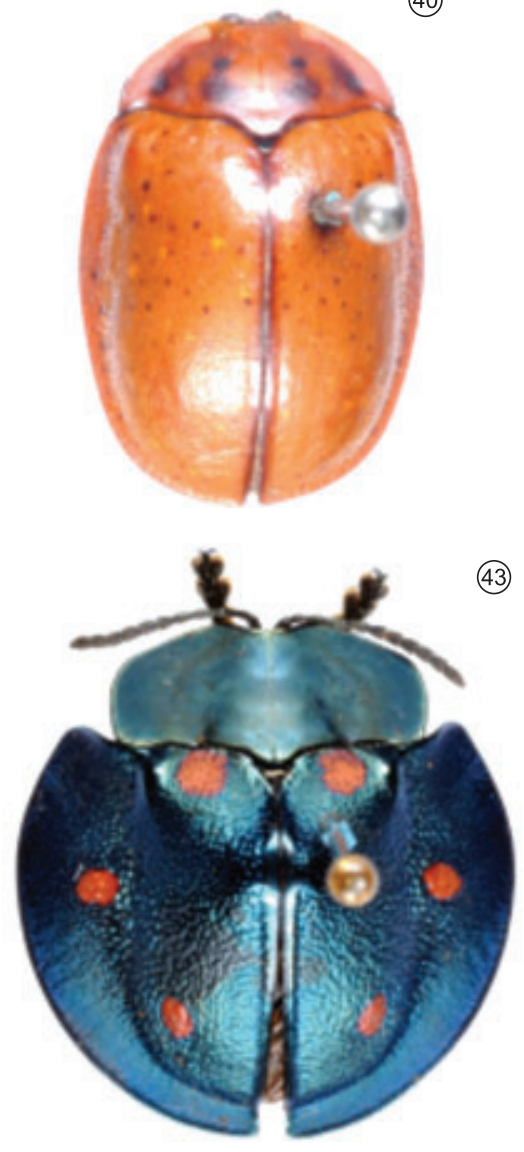

(43)
(38)

(39)
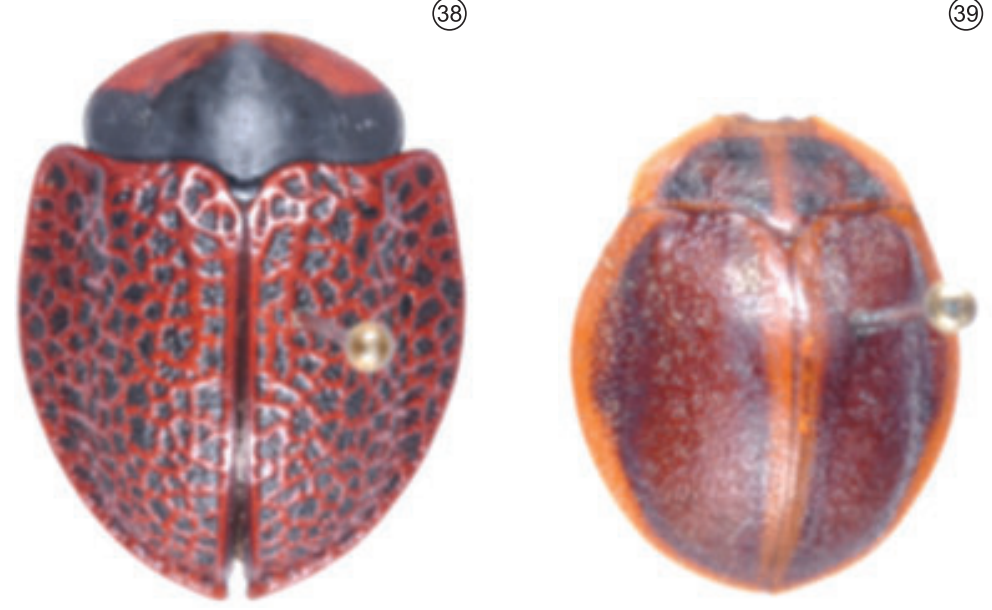

(41)
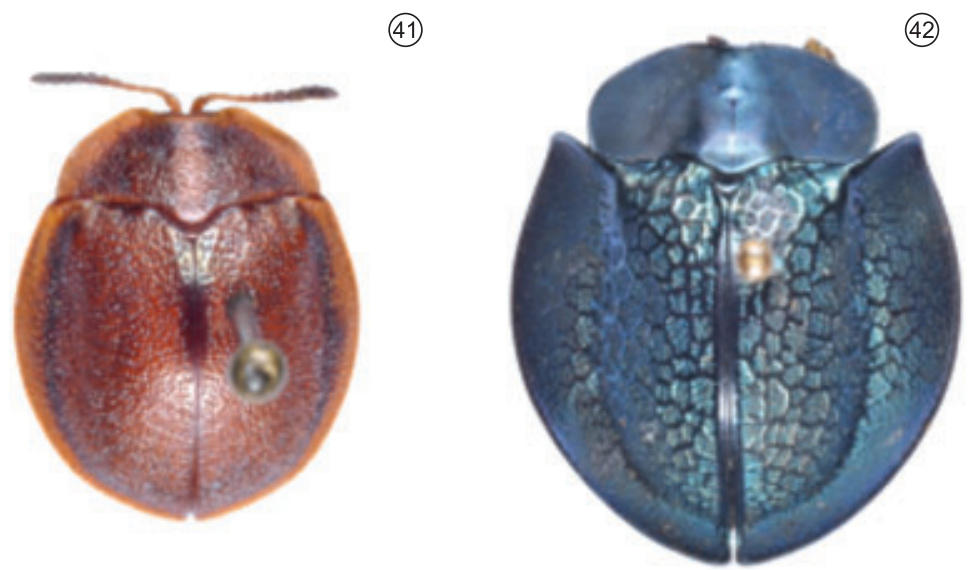

(45)
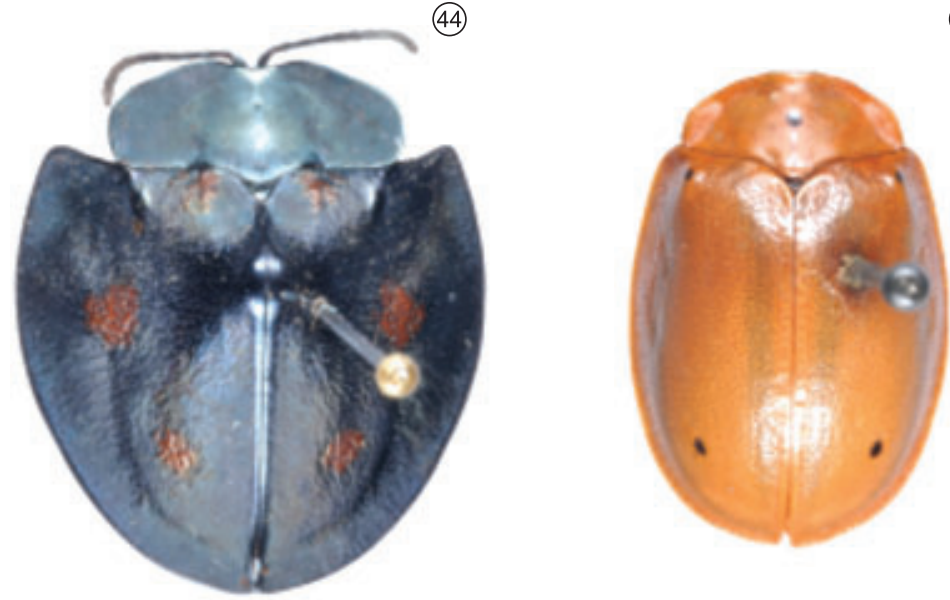

Figuras 37-45. 37) Anacassis fuscatta; 38) Botanochara impressa; 39) Chelymorpha clathrata; 40) Chelymorpha infecta; 41) Chelymorpha marginata; 42) Cyrtonota cyanea; 43) Cyrtonota sexpustulata; 44) Cyrtonota similata; 45) Hilarocassis albida.

Figures 37-45. 37) Anacassis fuscatta; 38) Botanochara impressa; 39) Chelymorpha clathrata; 40) Chelymorpha infecta; 41) Chelymorpha marginata; 42) Cyrtonota cyanea; 43) Cyrtonota sexpustulata; 44) Cyrtonota similata; 45) Hilarocassis albida.

Distribuição-Brasil (Minas Gerais, Rio de Janeiro, São Paulo) e Paraguai.

70. Mesomphalia sexmaculata (Boheman 1850) (Figura 46)

Mesomphalia sexmaculata Boheman 1850: 232.

Mesomphalia sexmaculata: Zikán \& Zikán 1967: 144, Flinte et al. 2009: 589.
Distribuição-Brasil (Minas Gerais, Rio de Janeiro, São Paulo).

71. Mesomphalia sublaevis (Boheman 1850)

Mesomphalia sublaevis Boheman 1850: 233.

Mesomphalia sublaevis: Flinte et al. 2009: 589.

Distribuição-Brasil (Rio de Janeiro, Rio Grande do Sul). 
72. Mesomphalia turrita (Illiger 1801)* (Figura 47)

Cassida turrita Illiger 1801: 180.

Mesomphalia turrita: Zikán \& Zikán 1967: 144, Flinte et al. 2009: 590. Distribuição-Brasil (Espírito Santo, Rio de Janeiro, São Paulo).
73. Omaspides pallidipennis (Boheman 1854) (Figura 48)

Omoplata pallidipennis Boheman 1854: 118.

Omaspides pallidipennis: Zikán \& Zikán 1967: 144, Flinte et al. 2009: 591.

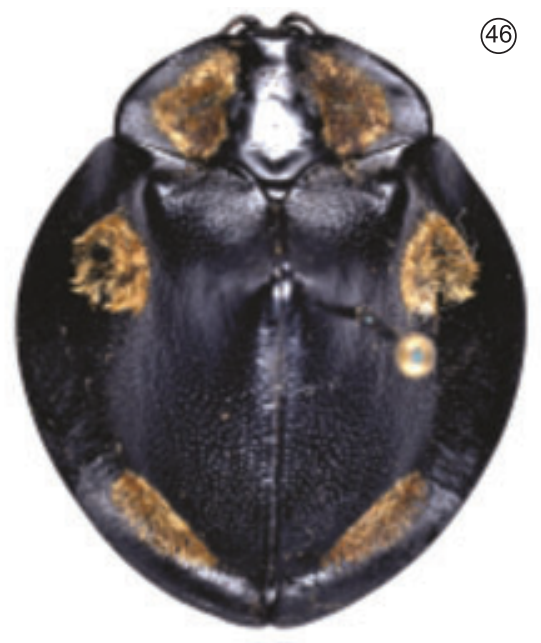

(49)

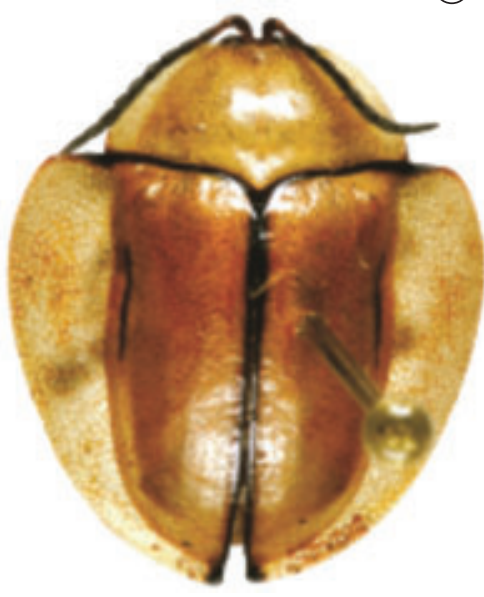

(52)

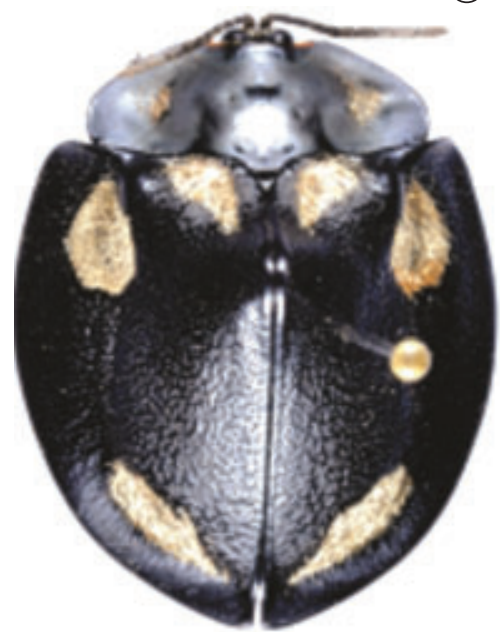

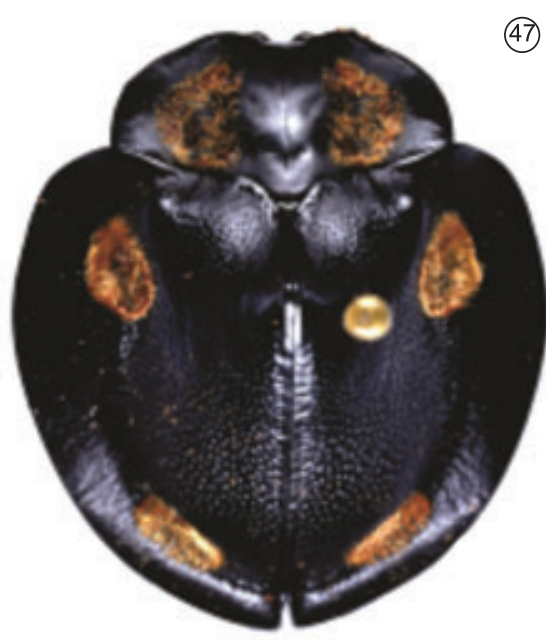

(47)

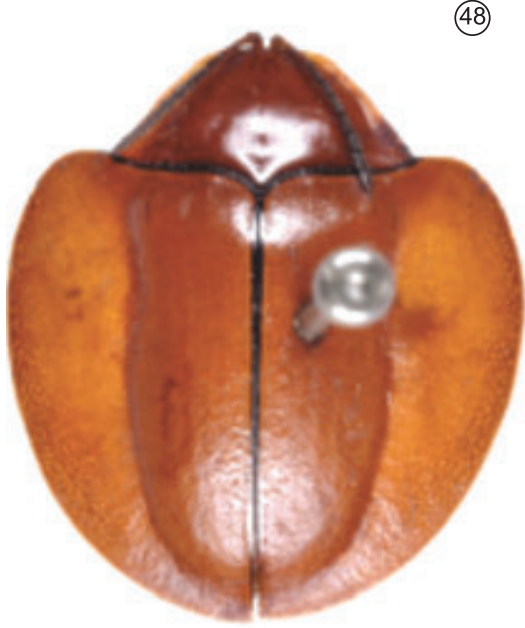

(50)

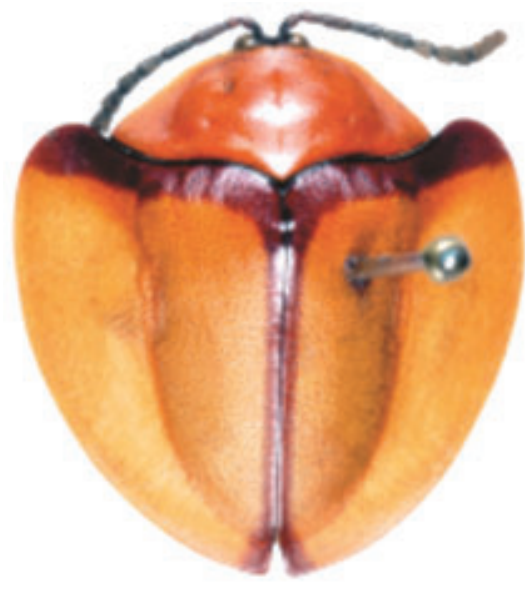

(53)

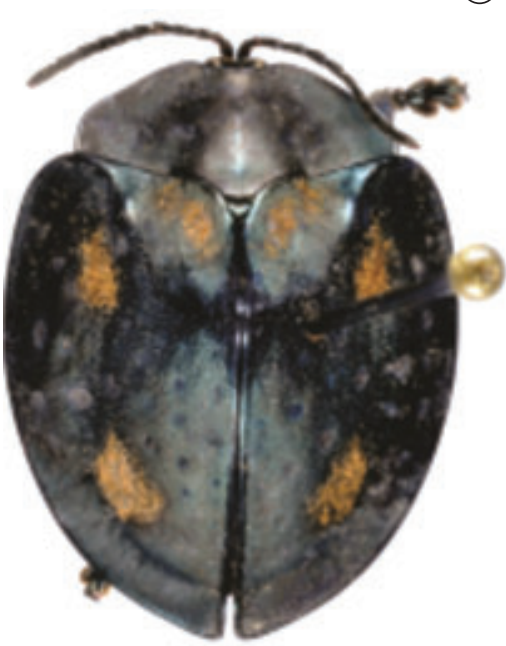

(54)

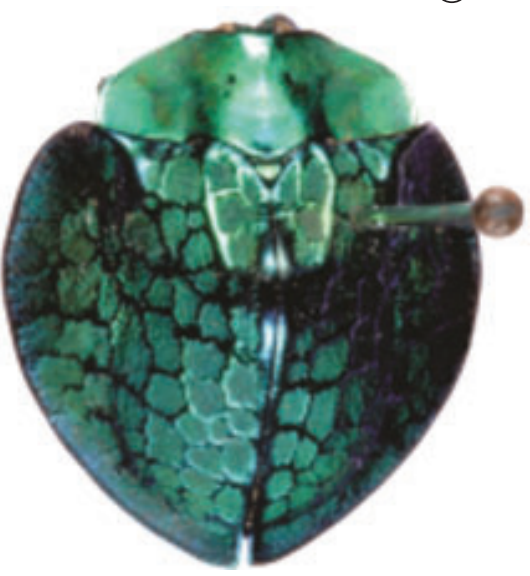

Figuras 46-54. 46) Mesomphalia sexmaculata; 47) Mesomphalia turrita; 48) Omaspides palidipennis; 49) Omaspides sobrina; 50) Paraselenis normalis; 51) Stolas areolata; 52) Stolas brevicuspis; 53) Stolas conspersa; 54) Stolas implexa. 
Distribuição-Brasil (Minas Gerais, Espírito Santo, São Paulo, Rio de Janeiro, Paraná, Santa Catarina).

74. Omaspides (Omaspides) trichroa (Boheman 1854)

Omoplata trichroa Boheman 1854: 117.

Omaspides (Omaspides) trichroa: Flinte et al. 2009: 592.

Distribuição-Guiana Francesa, Brasil (Rio de Janeiro), Bolívia e Peru.

75. Omaspides (Paromaspides) sobrina (Boheman 1854)*(Figura 49)

Omoplata sobrina Boheman 1854: 133.

Paromaspides sobrina: Zikán \& Zikán 1967: 144.

Omaspides (Paromaspides) sobrina: Flinte et al. 2009: 592.

Distribuição-Brasil (Bahia, Minas Gerais, Rio de Janeiro, Paraná) e Argentina.

76. Omaspides (Paramaspides) squalida (Boheman 1854)

Omoplata squalida Boheman 1854: 130.

Omaspides (Paramaspides) squalida: Flinte et al. 2009: 592.

Distribuição-Brasil (Rio de Janeiro, São Paulo).

77. Paraselenis axillaris (Sahlberg 1823)

Cassida axillaris Sahlberg 1823: 57.

Paraselenis axillaris: Flinte et al. 2009: 594.

Distribuição-Brasil (Mato Grosso, Minas Gerais, Rio de Janeiro).

78. Paraselenis (Spaethichoma) normalis (Germar 1824) (Figura 50)

Cassida normalis Germar 1824: 538.

Paraselenis (Spaethichoma) normalis: Flinte et al. 2009: 594.

Distribuição-Brasil (Minas Gerais, Rio de Janeiro).

79. Stolas aenea (Olivier 1790)

Cassida aenea Olivier 1790: 391.

Stolas aenea: Flinte et al. 2009: 598.

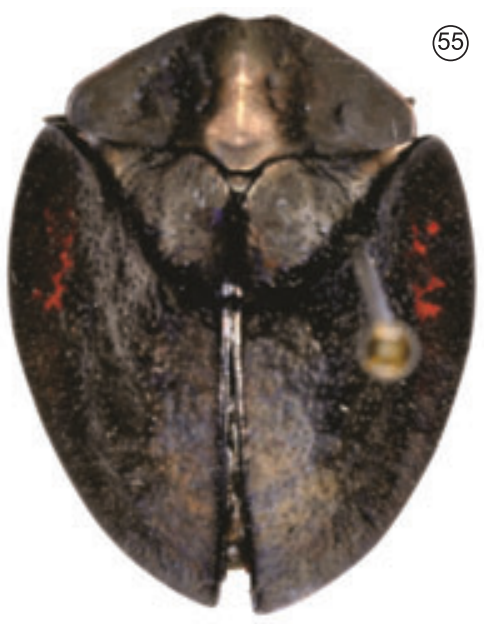

Distribuição-Guiana Francesa, Brasil (Minas Gerais, Rio de Janeiro, Santa Catarina) e Paraguai.

80. Stolas antiqua (Sahlberg 1823)

Cassida antiqua Sahlberg 1823: 56.

Stolas antiqua: Flinte et al. 2009: 598.

Distribuição-Brasil (Mato Grosso, Rio de Janeiro ao Rio Grande do Sul), Paraguai e Argentina.

81. Stolas areolata (Germar 1824)* (Figura 51)

Cassida areolata Germar 1824: 533.

Stolas areolata: Zikán \& Zikán 1967: 145, Flinte et al. 2009: 598.

Distribuição-Brasil (Minas Gerais, Rio de Janeiro ao Paraná) e Argentina.

82. Stolas brevicuspis (Spaeth 1922) (Figura 52)

PseudoMesomphalia brevicuspis Spaeth 1922: 174.

Stolas brevicuspis: Flinte et al. 2009: 598.

Distribuição-Brasil (Rio de Janeiro, São Paulo). Amplia-se a distribuição no Brasil para o Estado de Santa Catarina.

Material examinado. BRASIL, Santa Catarina: Corupá, 1 exemplar, A. Maller col. (MNRJ).

83. Stolas conspersa (Germar 1824) (Figura 53)

Cassida conspersa Germar 1824: 534.

Stolas conspersa: Zikán \& Zikán 1967: 145, Flinte et al. 2009: 599.

Distribuição-Brasil (Pará, Mato Grosso, Distrito Federal, Minas Gerais, Espírito Santo a São Paulo) e Paraguai.

84. Stolas implexa (Boheman 1850) (Figura 54)

Mesomphalia implexa Boheman 1850: 260.

Stolas implexa: Flinte et al. 2009: 599.

Distribuição-Brasil (Minas Gerais, Rio de Janeiro a Santa Catarina) e Uruguai.

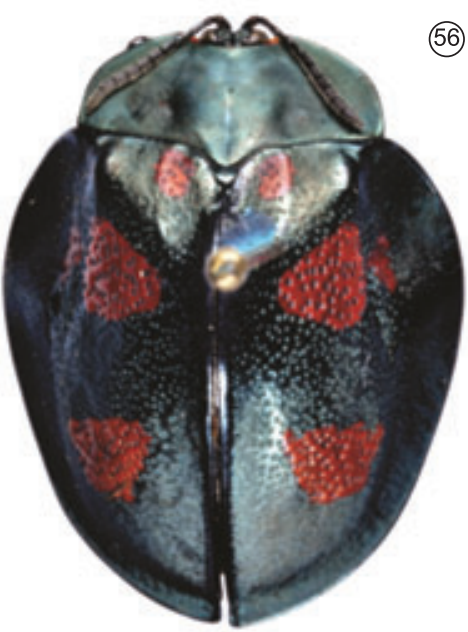

Figuras 55-56. 55) Stolas modica; 56) Stolas sexplagiata.

Figures 55-56. 55) Stolas modica; 56) Stolas sexplagiata. 
Simões, M.V.P. \& Monné, M.L.

85. Stolas impluviata (Boheman 1850)

Mesomphalia impluviata Boheman 1850: 255.

Stolas impluviata: Flinte et al. 2009: 599.

Distribuição-Brasil (Rio de Janeiro, Santa Catarina).

86. Stolas modica (Boheman 1850)* (Figura 55)

Mesomphalia modica Boheman 1850: 293.

Stolas modica: Flinte et al. 2009: 600.

Distribuição-Brasil (Bahia, Espírito Santo a São Paulo, Santa Catarina).

87. Stolas oblita (Boheman 1850)

Mesomphalia oblita Boheman 1850: 259.

Stolas oblita: Zikán \& Zikán 1967: 145.

Distribuição-Brasil (Pará, São Paulo, Rio de Janeiro, Santa Catarina).

88. Stolas sexplagiata (Boheman 1850) (Figura 56)

Mesomphalia sexplagiata Boheman 1850: 298.

Stolas sexplagiata: Flinte et al. 2009: 601.

Distribuição-Brasil (São Paulo, Rio de Janeiro).

\section{Agradecimentos}

Ao CNPq pela bolsa de iniciação científica n ${ }^{\text {o: }}$ 131504/2010-0 e ao IBAMA pela autorização de coleta n ${ }^{\circ}$ 16928-1. Ao Diretor de pesquisa do PNI, Leo Nascimento, por incentivar a realização dos estudos no parque. Ao Prof. Miguel A. Monné pelas sugestões ao manuscrito.

\section{Referências Bibliográficas}

BOHEMAN, C.H. 1850. Monographia Cassididarum. Tomus primus.ex Officina Norstedtiana, Holmiae, $452 \mathrm{p}$.

BOHEMAN, C.H., 1854. Monographia Cassididarum. Tomussecundus.ex Officina Norstedtiana, Holmiae, 506p.

BOHEMAN, C.H. 1855. Monographia Cassididarum. Tomus tertius.ex Officina Norstedtiana, Holmiae, 543p.

BOHEMAN, C.H. 1862. Monographia Cassididarum. Tomusquartus. exOfficina Norstedtiana, Holmiae, 504p.

BOROWIEC, L. \& WIETOJANSKA, J. 2010. World catalog of Cassidinae. $<$ http//www.biol.uni.wroc.pl/Cassidinae/catalog\%20internetowy/index. htm> (último acesso em 09/09/2010).

BURMEISTER, H. 1870. Cassidina Argentina. Ammerkungenzu Boheman's Monographia Cassididarum, die Artendes La Plata-Gebietesbetrehend. Stettin Ent. Ztg. 31:273-281.

CHABOO, C.S. 2007. Biology and phylogeny of the Cassidinae Gyllenhal sensu lato (tortoise and leaf-mining beetles) (Coleoptera: Chrysomelidae). Bull. Amer. Mus. Nat. Hist. 305:1-250. http://dx.doi.org/10.1206/00030090(2007)305[1:BAPOTC]2.0.CO;2

COSTA LIMA, A. 1955. Insetos do Brasil. Escola Nacional de Agronomia, Rio de Janeiro, v.9, part.3, 289 p. Série Didática, n.11.
FABRICIUS, J.C. 1775. Systema Entomologiae, sistens Insectorum classes, ordines, genera, species, adiectissynonymis, locis, descriptionibus, observationibus. Officina Libraria Kortii, Flensburgiet Lipsiae, p.1-832. http://dx.doi.org/10.5962/bhl.title.36510

FABRICIUS, J.C. 1781. Species Insectorumeorum differentias specificas, synonymaauctorum, loca natalia, metamorphosis adiectis observationibus, descriptionibus. Carl Ernst Bohn., Hamburgi et Kilonii, 552p.

FLINTE, V., BOROWIEC, L., FREITAS, S., VIANA, J.H., FERNANDES, F.R., NOGUEIRA-DE-SÁ, F. MACEDO, M.V. \& MONTEIRO, R.F. 2009. Tortoise beetles of the State of Rio de Janeiro, Brazil (Coleoptera: Chrysomelidae: Cassidinae). Genus 20(4):571-614.

GERMAR, E.F. 1824. Insectorum species novae autminus cognitae, descriptionibus illustratae. Hendel \& Sons, Halae, 824p.

GUÉRIN-MÉNEVILLE, F.E. 1844. Iconographie du regne animal de G. Cuvier, on représention d'après nature de l'une des especès les plus remarquables et souvent non encore figurées de chaque genre d'animaux, pouvant servir d'atlas a tous les traités de zoologie. Baillière, Paris, v.7, $111 \mathrm{p}$.

ILLIGER, K. 1801. Neue Insekten. Mag. Insektenk. 1(1-2):163-208.

INSTITUTO BRASILEIRO DO MEIO AMBIENTE E DOS RECURSOS NATURAIS RENOVÁVEIS-IBAMA. http://www.icmbio.gov.br/ parna_itatiaia/ (último acesso em 16/11/2010).

KLUG, K. 1829. Preis-Verzeichniss vorrathiger Insecten-doubletten des Königl. Zoologischen Museums der Universität. Berlin, p.18.

LINNAEUS, C. 1758. Systema naturae per regne tria naturae secundum classes, ordines, genera, species, cum characteribus, differentiis, synonymis, locis. Salvius, Holmiae, p.823.

LINNAEUS, C. 1763. Amoenitates Academicae seu Dissertation esvariae Physicae, Medicae Botanicae antehac seorsim editae, nunc collectae etauctae cum tabulis aeneis. Salvius, Holmiae, v.6, p.486.

OLIVIER, A.G. 1790. In Encyclopédie méthodique: histoire naturelle, insectes. Panckoucke Libr. Liège. 5(1):1-368.

PANZER, G.W. 1798. Voets Beschreibungen und Abbildungen hartschaaligter Insecten, Coleoptera Linn. Aus dem Original getreu übersetzt, mit der in selbigem fehlenden Synonymie und beständigem Commentar versehen von D. Georg Wolfgang Franz Panzer.Erlangen, Palm, p.1-112.

SAHLBERG, C.R. 1823. Periculi entomographici, species insectorum, nondum descriptas propositur. Aboae, p.1-82. http://dx.doi.org/10.5962/ bhl.title. 10471

SPAETH, F. 1902. Neue Cassidenaus Peru. Deutsche Ent. Zeitschr.1:81-102.

SPAETH, F. 1909. Kritische Studien über Chelymorpha Boh. und die verwandten Gattungen (Col.). Deutsche Ent. Zeitschr. 1909:715-732.

SPAETH, F. 1913. Kritische Studien über den Umfang und die Begrenzung mehrerer Cassiden-Gattungen nebst Beschreibung neuer amerikan. Arten. Archiv. F. Naturges. 79:126-164.

SPAETH, F. 1922. Neue Cassidinen (Coleoptera) des tropischen Amerika aus meiner und der Sammlung des zoologisk Museum in Kristiania. Norsk Ent. Tidsskr. 1:165-181.

SPAETH, F. 1926. Beschreibung neuer Cassidinen. Bull. Mens. Soc. Nat. Luxemb. 20:11-24, 47-60.

SPAETH, F. 1932. Neue Cassidinen (Col. Chrysom.). Stettiner. Ent. Zeitung. 93:182-204.

SWEDERUS, N.S. 1787. Et nytt genus, och femtio nya species af Insecter beskrifne. K. Svenska Vetens Akad. Hand. 8:181-290.

ZIKÁN, J.F. \& ZIKÁN, W.A. 1967. Inseto-Fauna do Itatiaia e da Mantiqueira. Revta Bras. Ent. 12:118-154.

Recebido em 16/11/2010

Versão reformulada recebida em 08/09/2011

Publicado em 18/11/2011 